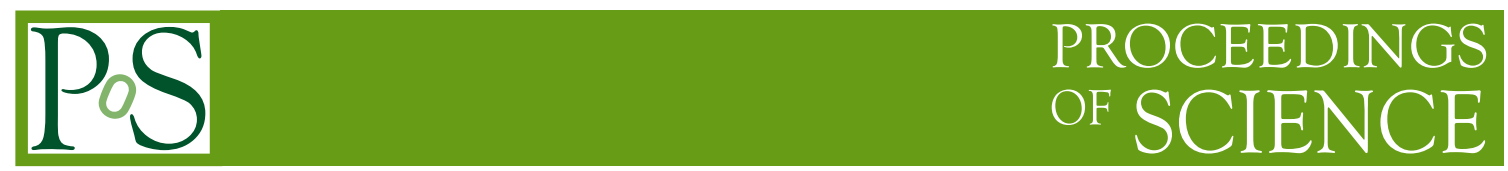

\title{
Long term stability analysis on the MD-A under TIBET III array
}

M. Amenomori ${ }^{1}$, X. J. Bi ${ }^{2}$, D. Chen ${ }^{3}$, T. L. Chen ${ }^{4}$, W. Y. Chen ${ }^{2}$, S. W. Cui ${ }^{5}$, Danzengluobu $^{4}$, L. K. Ding ${ }^{2}$, C. F. Feng ${ }^{6}$, Zhaoyang Feng ${ }^{2}$, Z. Y. Feng ${ }^{7}$, Q. B. Gou ${ }^{2}$, Y. Q. Guo ${ }^{2}$, H. H. He ${ }^{2}$, Z. T. He ${ }^{5}$, K. Hibino ${ }^{8}$, N. Hotta ${ }^{9}$, Haibing $\mathrm{Hu}^{4}$, H. B. $\mathrm{Hu}^{2}$, J. Huang ${ }^{2}$, H. Y. Jia ${ }^{7}$, L. Jiang ${ }^{2}$, F. Kajino ${ }^{10}$, K. Kasahara ${ }^{11}$, Y. Katayose ${ }^{12}$, C. Kato ${ }^{13}$, K. Kawata ${ }^{14}$, M. Kozai ${ }^{13}$, Labaciren ${ }^{4}$, G. M. Le ${ }^{15}$, A. F. Li ${ }^{16,6,2}$, H. J. Li ${ }^{4}$, W. J. Li ${ }^{2,7}$, C. Liu $^{2}$, J. S. Liu ${ }^{2}$, M. Y. Liu ${ }^{4}$, H. Lu ${ }^{2}$, X. R. Meng ${ }^{4}$, T. Miyazaki ${ }^{13}$, K. Mizutani ${ }^{11,17}$, K. Munakata ${ }^{13}$, T. Nakajima ${ }^{13}$, Y. Nakamura ${ }^{13}$, H. Nanjo ${ }^{1}$, M. Nishizawa ${ }^{18}$, T. Niwa ${ }^{13}$, M. Ohnishi ${ }^{14}$, I. Ohta ${ }^{19}$, S. Ozawa ${ }^{11}$, X. L. Qian ${ }^{* 6,2}$, X. B. Qu ${ }^{20}$, T. Saito ${ }^{21}$, T. Y. Saito ${ }^{22}$, M. Sakata ${ }^{10}$, T. K. Sako ${ }^{14}$, J. Shao ${ }^{2,6}$, M. Shibata ${ }^{12}$, A. Shiomi ${ }^{23}$, T. Shirai ${ }^{8}$, H. Sugimoto ${ }^{24}$, M. Takita ${ }^{14}$, Y. H. Tan ${ }^{2}$, N. Tateyama ${ }^{8}$, S. Torii ${ }^{11}$, H. Tsuchiya ${ }^{25}$, S. Udo ${ }^{8}$, H. Wang ${ }^{2}$, H. R. Wu ${ }^{2}$, L. Xue ${ }^{6}$, Y. Yamamoto ${ }^{10}$, K. Yamauchi ${ }^{12}$, Z. Yang ${ }^{2}$, S. Yasue ${ }^{26}$, A. F. Yuan ${ }^{4}$, T. Yuda ${ }^{14}$, L. M. Zhai ${ }^{2}$, H. M. Zhang ${ }^{2}$, J. L. Zhang ${ }^{2}$, X. Y. Zhang ${ }^{6}$, Y. Zhang ${ }^{2}$, Yi Zhang ${ }^{2}$, Ying Zhang ${ }^{2}$, Zhaxisangzhu ${ }^{4}$, X. X. Zhou ${ }^{7}$ 


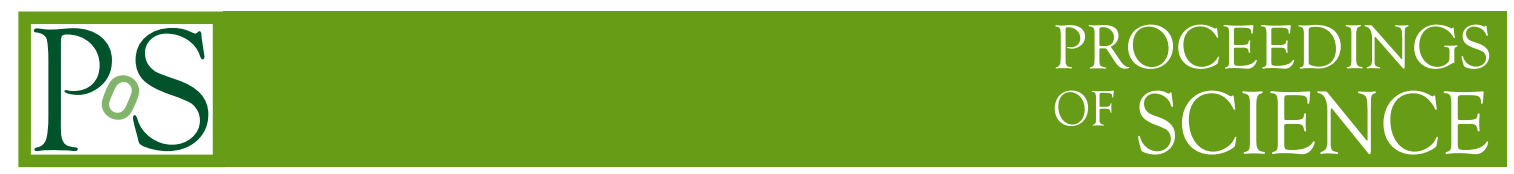

(The Tibet AS $\gamma$ Collaboration)

${ }^{1}$ Department of Physics, Hirosaki University, Hirosaki 036-8561, Japan

${ }^{2}$ Key Laboratory of Particle Astrophysics, Institute of High Energy Physics, Chinese Academy of Sciences, Beijing 100049, China

${ }^{3}$ National Astronomical Observatories, Chinese Academy of Sciences, Beijing 100012, China

${ }^{4}$ Department of Mathematics and Physics, Tibet University, Lhasa 850000, China

${ }^{5}$ Department of Physics, Hebei Normal University, Shijiazhuang 050016, China

${ }^{6}$ Department of Physics, Shandong University, Jinan 250100, China

${ }^{7}$ Institute of Modern Physics, SouthWest Jiaotong University, Chengdu 610031, China

${ }^{8}$ Faculty of Engineering, Kanagawa University, Yokohama 221-8686, Japan

${ }^{9}$ Faculty of Education, Utsunomiya University, Utsunomiya 321-8505, Japan

${ }^{10}$ Department of Physics, Konan University, Kobe 658-8501, Japan

${ }^{11}$ Research Institute for Science and Engineering, Waseda University, Tokyo 169-8555, Japan

${ }^{12}$ Faculty of Engineering, Yokohama National University, Yokohama 240-8501, Japan

${ }^{13}$ Department of Physics, Shinshu University, Matsumoto 390-8621, Japan

${ }^{14}$ Institute for Cosmic Ray Research, University of Tokyo, Kashiwa 277-8582, Japan

${ }^{15}$ National Center for Space Weather, China Meteorological Administration, Beijing 100081, China

${ }^{16}$ School of Information Science and Engineering, Shandong Agriculture University, Taian 271018, China

${ }^{17}$ Saitama University, Saitama 338-8570, Japan

${ }^{18}$ National Institute of Informatics, Tokyo 101-8430, Japan

${ }^{19}$ Sakushin Gakuin University, Utsunomiya 321-3295, Japan

${ }^{20}$ College of Science, China University of Petroleum, Qingdao 266555, China

${ }^{21}$ Tokyo Metropolitan College of Industrial Technology, Tokyo 116-8523, Japan

${ }^{22}$ Max-Planck-Institut für Physik, München D-80805, Deutschland

${ }^{23}$ College of Industrial Technology, Nihon University, Narashino 275-8576, Japan

${ }^{24}$ Shonan Institute of Technology, Fujisawa 251-8511, Japan

${ }^{25}$ Japan Atomic Energy Agency, Tokai-mura 319-1195, Japan

${ }^{26}$ School of General Education, Shinshu University, Matsumoto 390-8621, Japan $\dagger$

E-mail: qianxl@ihep.ac.cn

The underground muon detector with water Cherenkov technique is constructed as the upgrade of the Tibet air shower array, aiming at a higher sensitivity for gamma-ray observation. In one of the modules (MD-A), the full-sealing large Tyvek bag is used as a closed container. As the MD-A has been operated for more than one year, the long term stability of the performance of such detector is reported.

The 34th International Cosmic Ray Conference,

30 July- 6 August, 2015

The Hague, The Netherlands

\footnotetext{
${ }^{*}$ Speaker.

$\dagger$ The collaborative experiment of the Tibet Air Shower Arrays has been performed under the auspices of the Ministry of Science and Technology of China and the Ministry of Foreign Affairs of Japan. This work is Supported by the National Natural Science Foundation of China (Nos. 11165013, 11135010, 11105156, 11405180, 11463004, 11375209), by the 973 Program of China(No. 2013CB837000), and by Youth Innovation Promotion Association, Chinese Academy of Sciences.
} 


\section{Introduction}

The observation of the very high energy (VHE) gamma rays has an important impact on the development of astrophysics, cosmology, and particle astrophysics. In the last decade, benefited from the great success of satellite experiments and ground-based imaging atmospheric Cherenkov telescopes (IACT), the study of VHE gamma ray astronomy achieved a tremendous progress. The IACT experiments have discovered more than 161 new $\mathrm{TeV}$ sources[1][2]; the space-borne detector of Fermi-LAT has discovered 3000 new gamma ray sources in the energy range from $100 \mathrm{MeV}$ to $300 \mathrm{GeV}[3]$.

Finding evidence for the acceleration of cosmic ray nuclei has long been a key issue in attempts to elucidate the origin of cosmic rays. A thrilling results reported by Fermi-LAT provides the direct evidence that cosmic-ray protons are accelerated in SNRs through detecting the characteristic piondecay feature in the gamma-ray spectra of two SNRs, IC443 and W44[4].

On the other hand, the observation of high energy gamma rays above $100 \mathrm{TeV}$ is crucial in disentangle the mystery of the Galactic cosmic rays origin. Cosmic ray nuclei may generate high energy gamma rays through the decay of $\pi^{0}$ generated in its hadronic interaction with ambient gas. The gamma ray spectrum from hadronic process inherits that of the cosmic ray and extends to PeV energy. The characteristic feature of an electron process should have a cut off at multi-TeV because of the Klein-Nishimia effect and energy loss of electron by its synchrotron radiation. The observation of gamma rays in $100 \mathrm{TeV}$ energy region becomes very important in searching for hadronic source.

Ground-based extensive air shower arrays (EAS) have large effective area, wide field of view and high duty cycle, which make it a great potential in exploring the gamma rays emission at 100 $\mathrm{TeV}$, of which the flux is quite low. Still, its ability to identify gamma rays from the primary cosmic rays needs to be improved, considering the fact that the background of cosmic rays is much more abundant than gamma rays. Hence, the underground muon detectors (MD) have been built under the Tibet Air Shower (AS) surface array using water Cherenkov technique. The upgraded Tibet air shower array would be an ideal experiment in observing $100 \mathrm{TeV}$ gamma rays providing it could reach the initial goal in distinguishing the gamma rays from the overwhelming cosmic ray background.

\section{The upgrade of AS $\gamma$ experiment}

The Tibet AS array has been in operation at Yangbajing $\left(90^{\circ} 31^{\prime} \mathrm{E}, 30^{\circ} 06^{\prime} \mathrm{N} ; 4300 \mathrm{~m}\right.$ above sea level) in Tibet, China since 1990. The original array mainly consisted of plastic scintillator detector. In the late fall of 2003, the area of the Tibet AS array was further enlarged up to 36,900 $m^{2}$ as TIBET III array which consists of 728 FT-counters (249 of them also have 1 D-PMT) and 28 D counters[5].

The TIBET III array has been working in a wide energy range from TeV to $100 \mathrm{PeV}$. For gamma rays at $100 \mathrm{TeV}$, the angular resolution is about 0.2 degrees and the energy resolution is about $40 \%$. The Tibet AS array has obtained many important physics results since its operation. The Tibet AS experiment is the one that succeeded in measuring the two dimensional high-precision largescale cosmic-ray anisotropy in the northern sky and pointing out a new component of anisotropy 


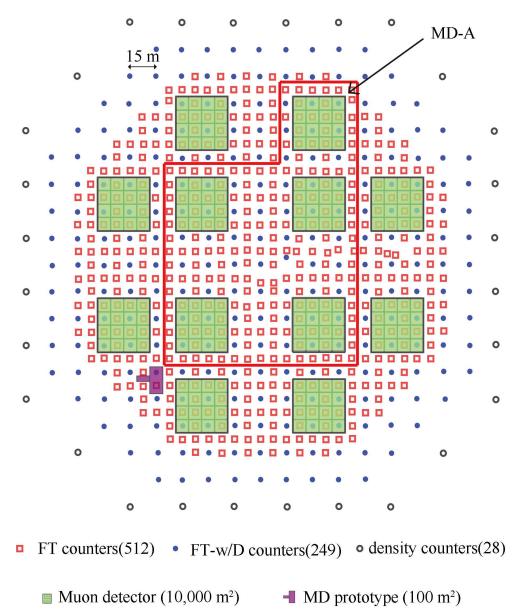

Figure 1: Schematic view of the Tibet III+MD array. Open squares: fast timing counters with a FT-PMT (FT counters); filled circles: FT counters with a D-PMT (FT-w/D counters); open circles: density counters with a D-PMT. The red box indicates MD-I (5 modules, $4500 \mathrm{~m}^{2}$ in total, 16 pools $(4 \times 4)$ each module), constructed in 2010. Module MD-A is located at the upper right corner of MD-I.

in the direction of Cygnus region at multi- $\mathrm{TeV}$ energies[6], where the discovery of $\mathrm{TeV}$ diffuse gamma-ray signal was soon claimed by Milagro[7].

To improve the sensitivity of gamma ray observation at about $100 \mathrm{TeV}$, we have planned to construct a $10000 \mathrm{~m}^{2}$ underground muon detector array using water Cherenkov technique. Together with the surface array, it constitutes as one important component of the new TIBET III+MD hybrid array[8][9]. Primary cosmic rays can produce a large number of muons in shower development, and the number and energy of the muons is proportional to the energy of the primary cosmic ray. Primary gamma rays and electrons produce much less muons than the cosmic ray. Therefore, the number of muons in an air shower can be used as a parameter to distinguish primary gamma rays from cosmic ray background. For a full-scale MD configuration[10], Monte Carlo (MC) simulation shows that nearly $99.99 \%$ of the cosmic ray background events can be rejected, while $99 \%$ of the primary gamma ray events at $100 \mathrm{TeV}$ retained, according to which, a background-free property would be realized at energy above $100 \mathrm{TeV}$.

In 2010, the MD-I ( 5 out of 12 modules) was constructed and the detector has been in operation since the fall of 2013. Figure 1 shows the schematic diagram of the existing Tibet III array and the muon detector. Each module has 16 units, and the volume of each unit is $7.2 \mathrm{~m} \times 7.2 \mathrm{~m} \times 2.4 \mathrm{~m}$. The detector is constructed $2.5 \mathrm{~m}$ below the ground in order to shield the electromagnetic particles produced by shower. The effective area of muon detector unit is 50 square meters, the spacing of two units center is $7.5 \mathrm{~m}$, which is corresponding with the spacing of the surface scintillator detector. Different from the other four modules, the top right module (MD-A) uses large Tyvek bag filled with high purity water[11]. One HAMAMATSU R3600 PMT is installed in each pool. Figure 2 shows the schematic view of one unit.

A single VME create readout scheme was developed for the MD-A data acquisition system. PC connects with the VME controller through Ethernet while the VME controller communicates with the electronics board through the back board bus. Generated from the MD-A detectors, there are 16 PMT signals in total. The electronics board collects the 16 signals and sends them to PC 




Figure 2: Schematic view of one pool in MD-A. Each pool is a water Cherenkov detector with $7.2 \mathrm{~m} \times 7.2 \mathrm{~m} \times 2.5 \mathrm{~m}$. One 20-inch PMT is mounted at the center of the ceiling and dip into the water to overlook the pool. The bag is filled with high-purity water to keep long-term stability of the water quality.

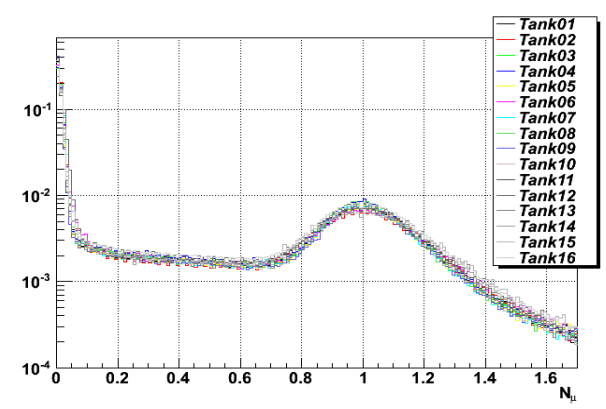

Figure 3: The single muon amplitude spectrum of 16 pools in MD-A.

by the VME controller. The trigger signal is provided by the Tibet AS array. When an air shower is detected by the surface detector array, a trigger signal is generated. The trigger rate of Tibet III array is about $1.7 \mathrm{kHz}$. Since December 2013, the MD-A data acquisition system has been in operating. The data acquisition system proves to be stable and reliable according to monitoring the system.

\section{The signal of single muon events}

The main goal of the upgrade of the Tibet AS experiment is to detect gamma rays at about 100 $\mathrm{TeV}$. About 10 muons are expected to be detected by the muon detector unit near the shower core of a $100 \mathrm{TeV}$ cosmic ray, so the dynamic range of MD-A is designed for about 15 muons. Figure 3 shows the single muon amplitude spectrum of 16 pools in MD-A. The discriminating threshold of the single muon amplitude spectrum is about 0.3 muon. Single muon peaks of 16 pools in Figure 3 show good consistency which indicates consistent properties of the 16 pools. Muon peak resolution (FWHM), a widely used measure of muon detector performance, in this experiment is around $50 \%$. According to the gain of each PMT, the number of the photoelectrons detected from MD-A unit is around 300 .

The MD-A electronic system record the charge and time information of muon from each detector. Using this information we can reconstruct the air shower event so that the direction, position 


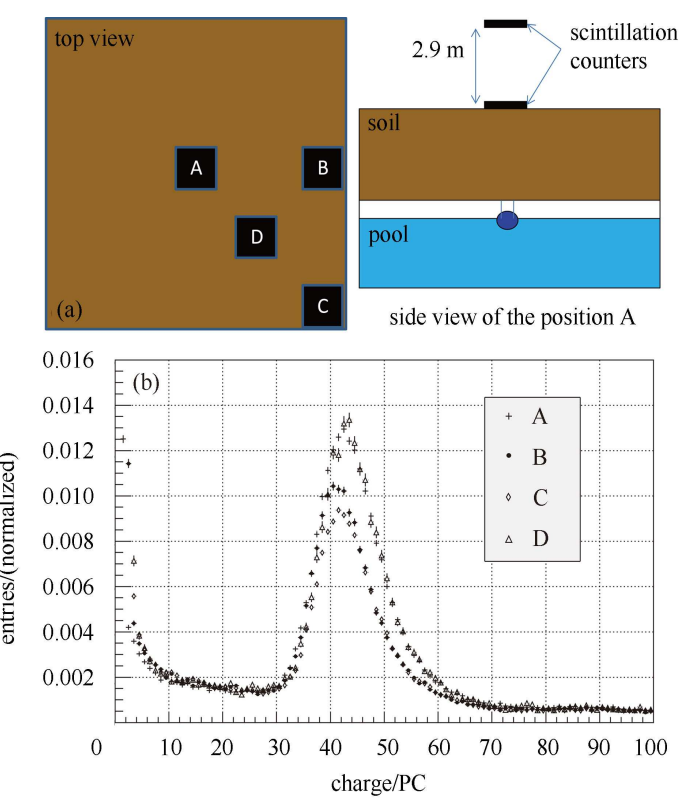

Figure 4: (a) The schematic view of different positions A, B, C and D; (b) The single muon amplitude spectrums for near vertical muon entering at Position A, B, C or D.

and energy information of air showers can be obtained. When the particle density is larger than 0.8 muon, the detector is "fired". In the reconstruction process, no less than 4 in 16 muon detectors are selected and used for fitting the plane of shower so that the shower direction can be reconstructed.

\section{Position non-uniformity}

The position non-uniformity is directly related to the muon number resolution of the detector, since we do not know the incident position in the pool for each muon. To study the position nonuniformity, four positions were selected as shown in Figure 4(a): the center of the pool (Position A), the side of the pool (Position B), the corner of the pool (Position C), the middle of A and C (Position D). The above-mentioned muon telescope was placed above the pool on Position A, B, C or D to select the vertical incident muons (the vertical spacing between two scintillation counters is $2.9 \mathrm{~m}$ ). Figure 4(b) shows the measured result. The obtained non-uniformity of the muon peak is better than $6 \%$.

\section{Summary}

Since the fall of 2013, MD-A detector and its data acquisition system has been in operation successfully. Experimental data have shown that the single muon peak can be identified clearly from noise. On the whole, the muon detector presents a high degree of consistency and good long-term stability. Further monitoring and study of the Tibet III MD-A is under way.

\section{References}

[1] Welcome to TeVCat. http://tevcat.uchicago.edu/. 
[2] The H.E.S.S. Source Catalog. http://www.mpi-hd.mpg.de/hfm/HESS/pages/home/sources/.

[3] F. Acero M. Ackermann M. Ajello et al. arXiv, 1501(02003).

[4] M. Ackermann et al. Science, 339:807-811, 2013.

[5] Amenomori M et al. Astrophys. J., 678:1165-1179, 2008.

[6] M. Amenomori. Science, 314:439-443, 2006.

[7] R. Atkins. PRL, 95(251103), 2005.

[8] Amenomori M et al. AIP Conf. Proc, 1085:723-726, 2008.

[9] Amenomori M et al. Journal of Physics, 120(062024), 2008.

[10] The Tibet AS $\gamma$ collaboration. 32nd ICRC, OG1.5(0351), 2011.

[11] Cheng Liu et al. Chinese Physics C, 37(026001), 2013. 